\title{
Placebo measurement: a new approach
}

\author{
Richard Morrisroe
}

School of Applied Psychology, UCC

"The question was a fashionable one, whether a definite line exists between psychological and physiological phenomena in human activity; and if so, where it lies?" -Tolstoy

\section{Background to the Placebo}

Placebos are at the same time one of the most mysterious and familiar experiences that we all have. Who amongst has not felt relieved as we swallow a painkiller, sure in the knowledge that it will help? Conversely, the placebo is mysterious in that it suggests that what we think and feel has a large influence on how our body works, and whether or not it works well.

The word placebo comes from a Psalm from the Bible, and it originally referred to people who mourned loudly and falsely at a funeral. Over the next few hundred years, the term placebo came to mean any medicine prescribed by a doctor more to please a patient than to actually cure them. Some authorities on the subject have claimed that until the $20^{\text {th }}$ century, all of medicine was the placebo effect.

Whatever the truth to that, the placebo was ignored until the experiences of an American surgeon, Henry Beecher on the beaches of Normandy during the final stages of World War 2 were to change all of that. Beecher had run out of morphine, and when asked by a nurse what they were to do, he told her to inject a solution of salt water into the patients who requested pain relief. Surprisingly, for both of them, the makeshift solution worked, and placebos came in from the cold.

Beecher never forgot this experience, and in 1955 he published an article in the Journal of the American Medical Association entitled "The Powerful Placebo". This article was a collection of other research reports where patients had improved after being given placebos. Although some researchers in the 1990's have argued that these findings were overblown, the article stimulated research into the placebo. The requirement in the US for clinical trials of all new medicines also spurred research into placebo, even if only as a control for the biologically based therapies.

Even still, many regarded the placebo as a myth, a psychological phenomenon which only influenced how people thought they felt rather than any objective measurement of bodily function. These ideas were shattered in 1979, when Levine and colleagues showed that some (but not all) placebo responses could be reversed by the administration of naloxone, 
a drug which antagonises the opioid receptors in the brain and body. The conclusion drawn from this was that even the mere knowledge that one was being given a treatment could activate the body's internal painkilling system (or endorphins).

\section{My Research}

Since that point, placebo research has proceeded in leaps and bounds, though not without its stumbles. However, even though we understand a lot about how placebos produce pain-relieving effects (and to a lesser extent in depression and other conditions), there is still no regular predictor of the placebo response. This is the gap in the literature which my project aims to fill.

Currently, placebos are predicted by asking participants to rate, on a 0-10 scale, how much benefit they expect to gain from a treatment. This does work, to a certain extent, in that higher ratings of benefits are expected to correlate with higher levels of pain relief from the treatment. However, it leaves about $90 \%$ of the variation in pain ratings unexplained, which is completely unacceptable. Furthermore, we have no current way of predicting who will respond to what placebo, which is a huge difficulty for doctors trying to improve care, researchers trying to study the effect and clinical scientists trying to demonstrate the worth of their new drug.

\section{The Implicit Association Test (IAT)}

The way in which I am going to get around this problem is simple. In the last decade psychologists have developed a measure called the Implicit Association Test (IAT) which can assess attitudes from the speed at which participants can classify words into two different categories when they are presented on a computer screen. For instance, an IAT might measure the associations between flowers and insects and pleasant and unpleasant words. In a typical trial, people would see the word "rose" come up on the screen and would need to classify it as a flower by pressing a key. The really interesting part comes when people combine the flower-insect words with the pleasant-unpleasant words. In this case one key is used whenever rose or a pleasant word is seen on the screen, and the other key is used when insect or an unpleasant word appears on the screen. The method then reverses the arrangement so that flower and unpleasant share the same key, while insect and pleasant go together. The idea is that the difference in reaction times between the flower-pleasant and flower-unpleasant conditions is a measure of how strongly one likes flowers (or insects, for that matter).

Now, while you may think that the flower-insect difference is somewhat irrelevant, the method is very flexible, and has been applied to racial attitudes, gender attitudes, attitudes towards political candidates or even between different brands of soft drinks or foods. In 
each of these domains, the method has shown its usefulness (although as per normal, there have been criticisms and debates over the methods and meaning of the results). There is a website which collects IAT data on many subjects (Project Implicit) and a quick internet search should allow you to complete an IAT yourself (which is undoubtedly the best way to understand the method).

\section{My Plan of Action}

The aim of my project is to develop an IAT which can predict the placebo effect. This sounds simple. However, the most critical part of any IAT is the words used in the program, as well as the distinction (i.e. the pleasant-unpleasant dimension discussed above), and this is the part which has taken most of the time (and its not completed yet). To do this, I have sent out health, optimism and mindfulness questionnaires to over 1000 UCC students (apparently being optimistic is bad for your health, at least in UCC), interviewed doctors, alternative therapists and students, and asked people to tell me who the people they associate most with health are. The data I got from the ranking exercise I have then put into a method called a Repertory Grid, which will allow me to determine which words and phrases to use to measure the placebo response (at least in theory).

Another goal of my research is to develop a better self report measure of placebo expectancies. I talked about the current measurements above (i.e., how much do you expect this treatment to help you) and how this is useful, but not enough. Over the past six months, I have developed a measure called the Treatment Credibility Questionnaire which can assess the amount of belief people have in different forms of treatments for pain. I tested this in two samples, and currently over 1800 people have completed the instrument. Data analysis is ongoing, but it appears from early results that the questionnaire does what it is supposed to.

Obviously, the only way to make sure a measure works is to test it, and my measures are no different. Starting in the Autumn, I will be testing my IAT and questionnaire using a placebo analgesia experiment. In this, I will assess the pain reduction following a stimulus and the extent to which it can be predicted by my new measures. This is the crown of my research, and as it comes closer I feel more and more excited at the prospect of finally putting my ideas to the test of reality.

\section{Conclusion}

In conclusion, my research is important in that it could teach us more about how we heal ourselves, how we can help others to heal, and how we can ensure that effective treatments are not rejected before getting to the hospital or pharmacy. I believe that this 
research has the possibility to substantially increasing our understanding of the placebo, which that is something that we all want.

Thanks to my supervisors Dr Zelda Di Blasi, School of Applied Psychology, UCC and Dr Dylan Evans, School of Medicine, UCC. Thanks also to all of my participants, past and future. 